Investigations

\title{
Design and Realization of Pre-Amplifier and Filters for on- Board Radar System
}

\author{
${ }^{1}$ N. Dinesh Kumar, ${ }^{2}$ R. Divya Ravali and ${ }^{3}$ Panchavati R. Srirekha \\ ${ }^{1}$ Vignan Institute of Technology and Science, Deshmukhi, Hyderabad 508284, India \\ ${ }^{2}$ Senior System Engineer at Infosys Ltd, India \\ ${ }^{3}$ Accounts Manager in HCl Infosystems, India
}

Article history

Received: 23-12-2014

Revised: 22-04-2015

Accepted: 30-10-2015

Corresponding Author: N. Dinesh Kumar

Vignan Institute of Technology

and Science, Deshmukhi,

Hyderabad 508284, India

E-mail: dinuhai@yahoo.co.in

\begin{abstract}
Today's warfare situation incorporates a considerable measure of innovative weaponry and every nation is attempting to outmaneuver the other utilizing high engineering, henceforth it is of most extreme criticalness to be at the front line of the bleeding edge innovation and having the best weapons conceivable to secure the country. One of the innovations which give the one having it the high ground is Radio Detection and Ranging (RADAR) engineering. Radar is an object recognition framework that uses electromagnetic waves to recognize the range, vehicles, climate developments and landscape. A radar framework incorporates a transmitter which radiates an arrangement of EM pulses. These pulses are transmitted in a directional pattern using a suitable highgain antenna. The radar then uses a collector, normally joined with the same radio wire, to catch any returned pulses being reflected from items showing up in the reception apparatus' field of perspective. The transmitter, antenna and recipient are connected utilizing a circulator. This guarantees that all the transmitter power is sent to the radio wire whilst all the reflected power came back to the reception apparatus. The echo signal coming back to the receiver is a high recurrence signal in Ghz extent having low adequacy. The processing of this sort of signal is extremely troublesome and henceforth it needs to be down changed over into an Intermediate Frequency (IF) signal whose reach is in Mhz. This down transformation is carried out utilizing a super heterodyne receiver. Most radar receivers use megahertz Intermediate Frequency (IF) with a value around 30 and 75 megahertz. Ground based radar is utilized for missile tracking framework to get its range and angular position to block and destroy. Precision of these radars is not sufficient to hit and crush, so the rocket itself is provided with onboard radar to track the target, block it buzzing around and pulverize. Radar signal handling of onboard radar is carried out in both simple and advanced modes, The latter mode of signal preparing is much simple to actualize changes and up gradation. But the IF signal before digitization needs to be amplified to get enough signal strength and filtered to select the desired. This paper exhibits the design and realization of an amplifier and filters to process the received signal at IF level of a locally available radar framework. MULTISIM is utilized to confirm the channel qualities and intensification variable.
\end{abstract}

Keywords: Amplifiers, Bandwidth, Filters, Gain, Multisim, On-board Radar

\section{Introduction}

Today's warfare situation incorporates a considerable measure of cutting edge weaponry and every nation is attempting to outmaneuver the other utilizing high engineering, henceforth it is of most extreme imperativeness to be at the front line of the forefront innovation and having the best weapons conceivable to secure the country (Stephens, 1996). One of the advances which give the one having it the high ground is 
Radio Detection and Ranging (RADAR) engineering. Radar is an Object recognition framework that uses electromagnetic waves to distinguish the range, vehicles, climate developments and landscape. A radar framework incorporates a transmitter which emits a series of EM pulses. These pulses are transmitted in a directional example utilizing a suitable high-gain antenna. The radar then uses a receiver, generally associated with the same receiving antenna, to distinguish any returned pulses being reflected from articles showing up in the reception apparatus' field of perspective. The transmitter, antenna and receiver are connected utilizing a circulator. This guarantees that all the transmitter force is sent to the antenna wire whilst all the reflected power came back to the reception apparatus is sent to the receiver.

\section{Approach and Methodology}

The reverberation signal coming back to the collector is a high frequency signal in Ghz extent having low adequacy. The handling of this sort of sign is exceptionally troublesome and thus it needs to be down changed over into an Intermediate Frequency (IF) signal whose extent is in Mhz. This down change is carried out utilizing a super heterodyne recipient. Most radar recipients use megahertz an Intermediate Frequency (IF) with a worth somewhere around 30 and 75 megahertz. The IF is delivered by blending a local oscillator signal with the approaching or incoming signal. The nearby oscillator (LO) is vital to effective operation and must be both tunable and extremely steady.

A super heterodyne recipient utilizes frequency blending or heterodyning to change over a received signal to an fixed IF, which can be more advantageously handled than the first radio bearer frequency. The super heterodyne receiver uses one or more LO and mixers to change over the echo to an IF that is helpful for filtering and transforming operations. This down changed over IF signal is not difficult to process for getting the obliged data about the object. The signal is initially prepared in simple space and afterward changed over into advanced signal for further preparing. Since echo signal is having low abundancy, after down changing over the signal, it is intensified using a preamplifier. This intensified signal is band constrained utilizing a band pass filter. A certain $\mathrm{dB}$ of gain (approximately 20 $\mathrm{dB}$ ) is to be maintained throughout the processing. Thus the band constrained signal is again amplified to compensate any losses that may happen amid the band pass filtering stage. The following stage is a low pass filter, which acts as an anti-aliasing filter.

To change over a low pass signal to digital format the first necessity is to example the greatness of the analog signal at sampling rate. These magnitudes are then changed over to digital words. On the off chance that the signal transfer speed is excessively incredible, there will be contortion, which is alluded to as associating bending. The filter which defines this data transmission has low pass characteristics. Its purpose is to prevent aliasing distortion and so is defined as an anti-aliasing filter. At that point in the wake of passing through a low pass channel before testing, the signal is given to an analog to digital converter for further processing in digital domain.

\section{On-Board Radars}

Radar meets expectations by a procedure of transmitting and getting a radio signal. The radar framework measures the time taken for the sign to be reflected from items encompassing it and after that methods the data to make a picture of the area. Contingent upon the quality of the radio signal, it is conceivable to get an exact picture of objects in an encompassing territory (Skolnik, 1990). Anyway, it can just ready to spot questions that are inside an observable pathway and it paints a consistent picture around you, with any encompassing objects rendered in a roundabout example focused around the breadth of the transmission. Depending upon the rate with which the gadget pivots, the picture upgrades each few seconds, which implies that there may be a deferral in getting the data.

The heart of the radar framework is the modulator. It produces all the vital timing pulses (triggers) for utilization in the radar and its related frameworks and guarantees that all subsystems of the radar framework work in an definite time relationship with one another and that the interims between pulses, as well as the pulses themselves, will be of the legitimate length. The duplexer will be basically an electronic switch that grants a radar framework to use a single antenna to both transmit and receive. The duplexer must unite the antenna to the transmitter and detach the reception apparatus from the antenna for the duration of the transmitted pulses. The switching time will be called receiver recovery time and must be extremely quick if close-in targets are to be located.

The antenna framework routes the pulse from the transmitter, transmits it in a directional beam, lifts up the returning echo and passes it to the receiver with a least losses. The antenna framework incorporates the reception apparatus, transmission lines and waveguide from the transmitter to the antenna and transmission lines and waveguide from the antenna to the collector. The beneficiary acknowledges the frail RF echoes from the antenna apparatus framework and routes them to the markers perceivable video signals. Since the radar frequencies will be exceptionally high and troublesome to enhance, a super-heterodyne receiver 
will be utilized to change over the echoes to a lower frequency, called intermediate frequency (IF), which will be simpler to amplify.

Exchanging the antenna in the middle of transmit and get modes exhibits one issue; guaranteeing that greatest utilization is made of the available energy is an alternate. The least complex arrangement is to utilize a change to exchange the antenna association from the receiver to the transmitter during the transmitted pulse and once more to the recipient amid the echo pulse. No viable mechanical switches are accessible that can open and close in a couple of microseconds. Consequently, electronic switches must be utilized. After transformation to the IF, the signal is enhanced in a few IF-enhancer stages. The vast majority of the increase of the collector is produced in the IF amplifier stages. The general transmission capacity of the beneficiary is regularly dictated by the transfer speed of the IF stages. Gain must be variable to give a steady voltage output for input signals of diverse amplitudes (Bao et al., 2010):

Design Specifications

Amplifier Specifications

The specifications for designing the amplifier were:

Input voltage: 0.125 to $0.15 \mathrm{v}$

Gain: $20 \mathrm{~dB}$

Output voltage: $1.8 \mathrm{~V}$

Filter Specifications Lower cutoff frequency: Upper cutoff frequency: $25.5 \mathrm{MHz}$

Band Pass Filter

Bandwidth: $5 \mathrm{MHz}$

Pass band ripple: $2 \mathrm{~dB}$

Center frequency: $28.5 \mathrm{MHz}$

Low Pass Filter

Cutoff frequency: $30.5 \mathrm{MHz}$

Pass band ripple: $2 \mathrm{~dB}$

\section{Realisation of Amplifier}

\section{Low Distortion Amplifier Chip}

The AD8009 is an ultrahigh speed current

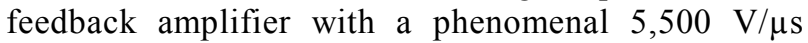
slew rate that results in a rise time of $545 \mathrm{ps}$, making it ideal as a pulse amplifier. The high slew rate reduces the effect of slew rate limiting and results in the large signal bandwidth. For applications with multi-tone signals, such as IF signal chains, the third order Intercept (3IP) of $12 \mathrm{dBm}$ is achieved at the same frequency. This distortion performance coupled with the current feedback architecture make the AD8009 a flexible component for a gain stage amplifier in IF/RF signal chains. The chip Pulse Amplifier is used in IF/RF Gain Stage/Amplifiers, High Resolution Video Graphics, High Speed Instrumentations and $\mathrm{CCD}$ Imaging Amplifier. The amplifier circuit shown in Fig. 1 is realized using MULTISIM based upon the desired specifications. The chip used is AD8009AR which has prerequisite features like large signal bandwidth, ultra high speed and low distortion over wide bandwidth etc.

The required gain with the two stages of amplifier is $20 \mathrm{~dB}$ with each stage providing a gain of $10 \mathrm{~dB}$ each. Table 1 shows the theoretical and simulated gain for single stage amplification for different values of feedback resistor. The simulation is done in MULTISIM and the gain is observed using spectrum analyzer.

Table 1. Gain of the amplifier for different values of $R_{f}$

\begin{tabular}{lllc}
\hline & & Gain $(\mathrm{dB})$ & \\
$R_{f}(\Omega)$ & $R_{2}(\Omega)$ & Theoretical & Simulated \\
\hline 200 & 100 & 9.542 & 9.549 \\
227 & 100 & 10.29 & 10.293 \\
332 & 100 & 12.709 & 11.750 \\
\hline
\end{tabular}

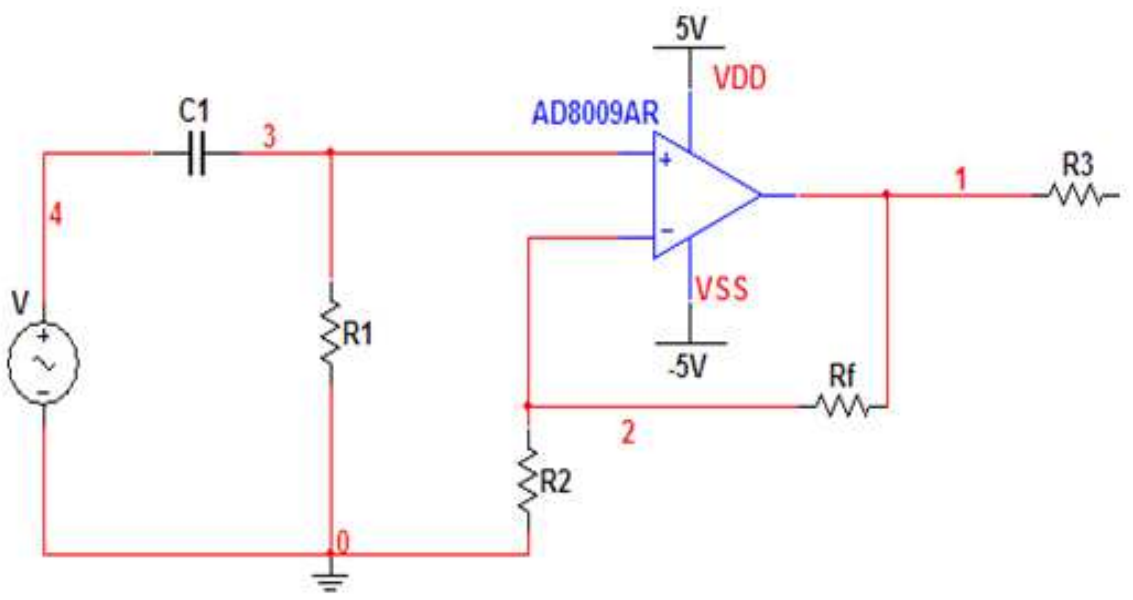

Fig. 1. Amplifier circuit 


\section{Design and Realisation of Analog Filters}

\section{Low Pass Filter}

The below Fig. 2 shows the circuit of a low pass filter. This circuit has to be simplified to get the transfer function and to relate it to some standard design like $\mathrm{m}$-derived, $\mathrm{T}$ or pi section etc. The final equivalent circuit is as shown in the Fig. 2 . The values of the impedances for the equivalent $\mathrm{T}$-section are as follows Equation 1:

$$
\begin{aligned}
& Z_{c}=\frac{\left(1-\omega^{2} L C\right)^{2}}{2 \omega^{2}\left(\begin{array}{l}
C_{1}-\omega^{2} L C C_{1}+2 C_{2} \\
-2 \omega^{2} L C C_{2}-2 \omega^{2} L C_{2}
\end{array}\right)\left(1-\omega^{2} L C+j \omega L C_{1}\right)} \\
& Z_{p}=\frac{L\left(1-\omega^{2} L C\right)\left(2 C_{2}+C_{1}\right)-2 \omega^{2} L^{2} C_{1} C_{2}}{\left[\begin{array}{l}
\left(1-\omega^{2} L C\right)\left(2 C_{2}+C_{1}\right) \\
-2 \omega^{2} L C_{2}
\end{array}\right]\left[1-\omega^{2} L C+j \omega L C_{1}\right]}
\end{aligned}
$$

The simulation of this filter gives the response as shown in the Fig. 3. It can be seen that the response is not up to the mark. The two main limitations of this response are no sharp cut off frequency at the required point and there is no proper attenuation in the stop band.

\section{Band Pass Filter}

The Fig. 4 shows the band pass filter circuit. This circuit has to be reduced to some standard design so that this circuit can be compared with that standard design. Hence, after this reduction the final circuit becomes an equivalent pi-section filter as shown $n$ Fig. 4 with impedances $Z_{p}, Z_{a}$ and $Z_{p}$. Where Equation 2 and 3:

$$
Z_{p}=\frac{j\left(\omega^{2} L C_{2}+\omega^{2} L C_{1}-1\right)\left\{\begin{array}{l}
2 \omega L C_{1}+\omega^{2} L\left(C_{1}+C_{2}+C_{3}\right) \\
-\omega^{4} L^{2}\left(2 C_{1} C_{2}+C_{2} C_{3}+C_{1} C_{3}\right)-1
\end{array}\right\}}{\left(C_{1}+C_{2}\right)\left\{\begin{array}{l}
\omega^{2} L\left(C_{1}+C_{2}+C_{3}\right) \\
-\omega^{4} L^{2}\left(2 C_{1} C_{2}+C_{2} C_{3}+C_{1} C_{3}\right)-1
\end{array}\right\}+2 \omega L C_{1} C_{2}}
$$

$$
Z_{a}=\frac{j\left(\omega^{2} L C_{2}+\omega^{2} L C_{1}-1\right)\left\{\begin{array}{l}
\left(\omega^{2} L C_{2}+\omega^{2} L C_{1}-1\right) \\
\left(1-\omega^{2} L C_{3}\right)-2 \omega^{4} C_{1} C_{2} L^{2} \\
+2 \omega^{2} L C_{1}
\end{array}\right\}}{\omega^{3} C_{1}^{2} L}
$$

From the response shown in Fig. 5, it can be observed that:

- Band width is more than required that is equal to $7.767 \mathrm{MHz}$ while the required bandwidth is only 5 $\mathrm{MHz}$, which means it allows undesired higher frequency components as well
- Attenuation is considerably less in the stop band

- Cutoff frequencies do not meet the specifications

\section{Design of Band Pass Filters}

To overcome the above said limitations, few filters have been designed which have better performance and response than the already designed filter.

\section{Design of Constant K-Type T-Section Filter}

The constant $\mathrm{k}$ or k-type filter section is the basic image filter section. It is the filter in which the series and shunt arm impedances $Z_{1}$ and $Z_{2}$ are connected by Equation 9:

$$
Z_{1} Z_{2}=R_{k}^{2}
$$

where, $R_{k}$ is a real constant that is a resistance independent of frequency. It is termed as design impedance or nominal impedance of the constant $\mathrm{K}$ filter. This filter has been designed because it is the simplest from the circuit topology point of view. The ktype also has moderately fast transition from the passband to the stopband and moderately good stopband rejection. The following are the steps involved for designing the constant k-type filter. Figure 6 is a constant k-type filter.

The anti resonant frequency of the shunt arm is made equal to the resonant frequency of the series arm Equation 4 and 5:

$\omega o^{2} L_{1} C_{1}=1=\omega o^{2} L_{2} C_{2} \Rightarrow L_{1} C_{1}=L_{2} C_{2}$

So from:

$$
L_{1} C_{1}=\frac{1}{\omega_{0}^{2}}
$$

The impedances of the arms are Equation 6 to 8:

$$
\begin{aligned}
& Z_{1}=j\left(\omega L_{1}-\frac{1}{\omega C_{1}}\right)=j\left(\frac{\omega^{2} L_{1} C_{1}}{\omega C_{1}}\right) \\
& Z_{2}=\frac{j \omega L_{2}\left(-j / \omega C_{2}\right)}{j\left(\omega L_{2}-1 / \omega C_{2}\right)}=\frac{j \omega L_{2}}{1-\omega^{2} L_{2} C_{2}}
\end{aligned}
$$

$$
Z_{1} Z_{2}=-\frac{L_{2}\left(\omega^{2} L_{2} C_{1}-1\right)}{C_{1}\left(1-\omega^{2} L_{2} C_{2}\right)}
$$

If $L_{1} C_{1}=L_{2} C_{2}$, then $Z_{1} Z_{2}=\frac{L_{2}}{C_{1}}=\frac{L_{1}}{C_{2}}=R_{k}{ }^{2}$ 

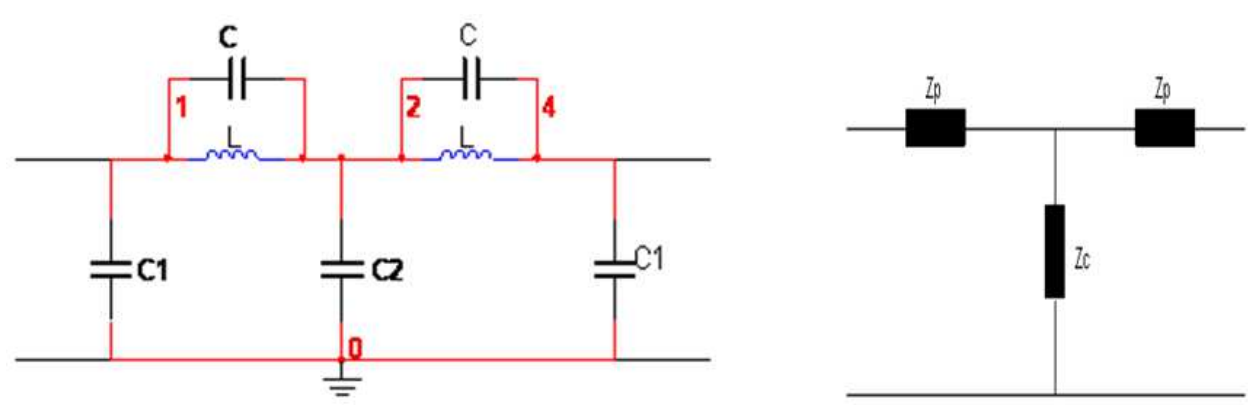

Fig. 2. Low pass filter circuit and equivalent T-network

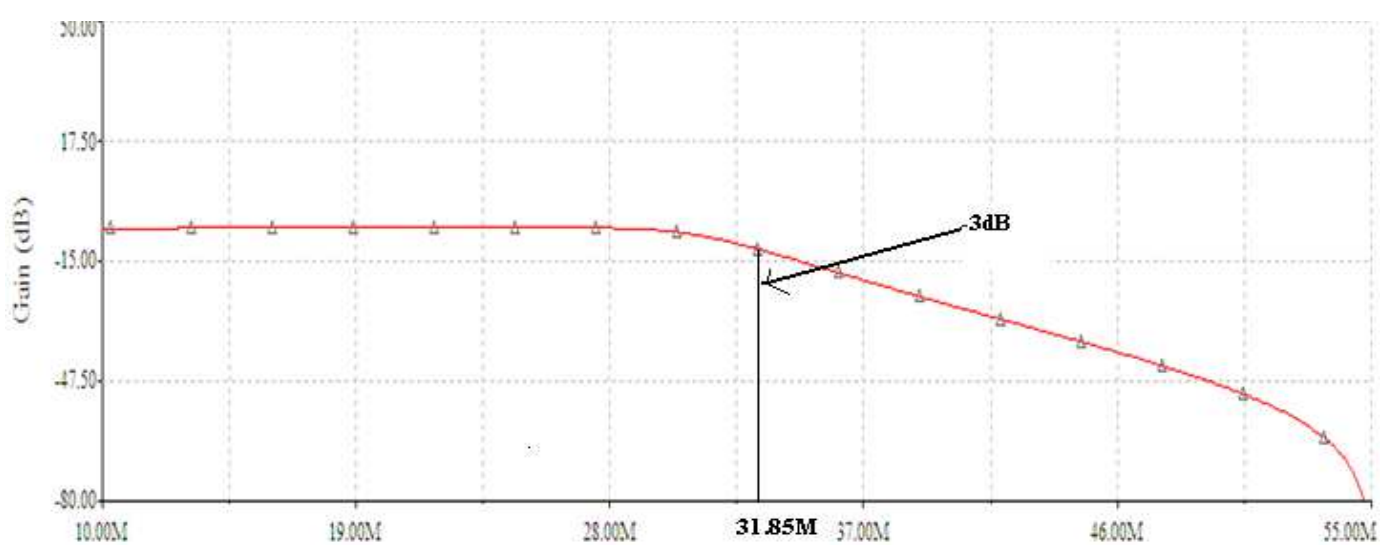

Fig. 3. Low pass filter response
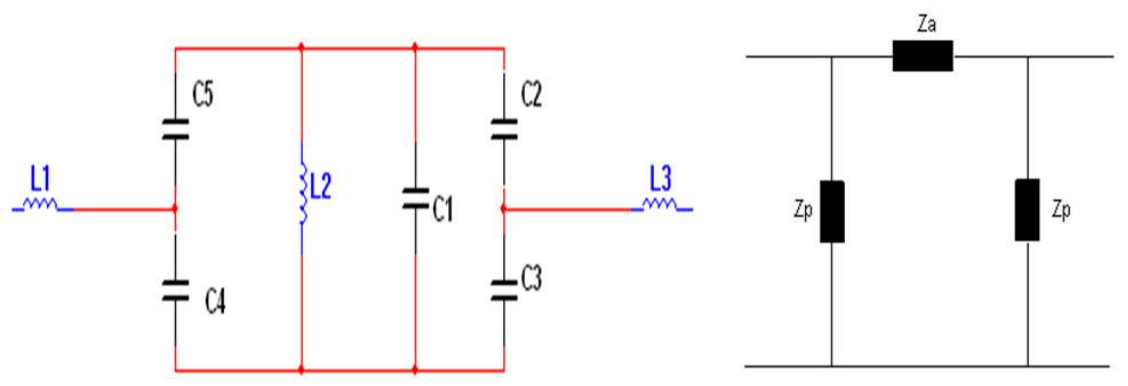

Fig. 4. Band pass filter and equivalent pi-network

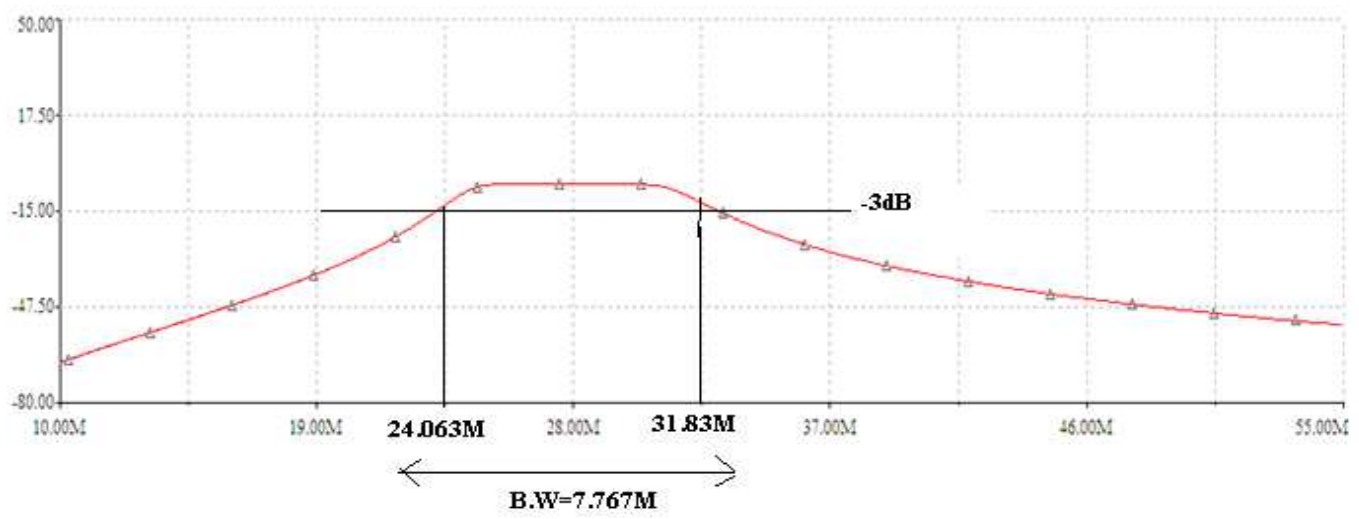

Fig. 5. Band pass filter response 


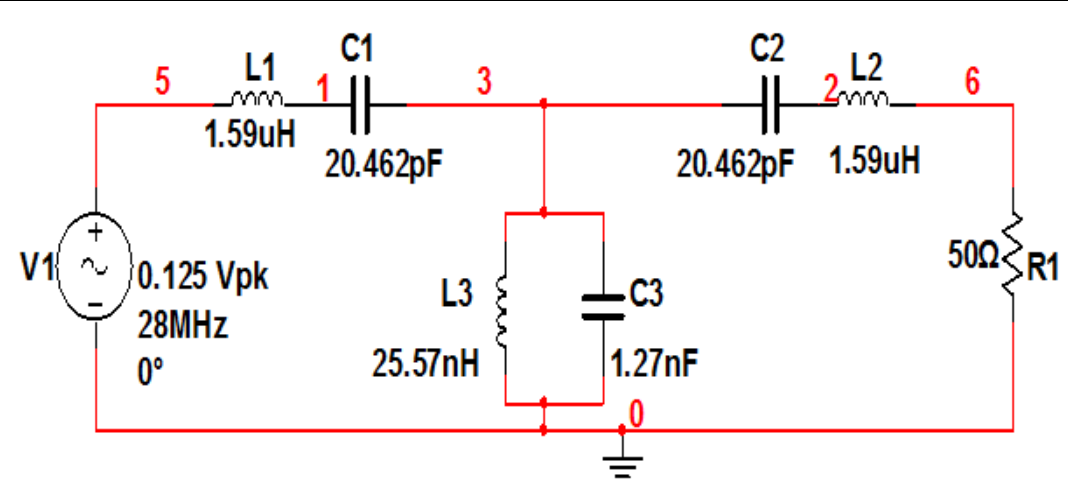

Fig. 6. Constant K-type band pass filter

At the cutoff frequencies Equation 10:

$$
\begin{aligned}
& Z_{1}=-4 Z_{2} \\
& Z_{1}^{2}=-4 Z_{1} Z_{2}=-4 R_{k}^{2} \\
& \Rightarrow Z_{1}= \pm j 2 R_{k}
\end{aligned}
$$

$Z_{1}$ at lower cutoff frequency $f_{1}=-Z_{1}$ at upper cutoff $f_{2}$.

The reactance of series arm at cutoff frequencies is Equation 11 and 12:

$$
\begin{aligned}
& \frac{1}{\omega_{1} C_{1}}-\omega_{1} L_{1}=\omega_{2} L_{1}-\frac{1}{\omega_{2} C_{1}} \\
& 1-\omega^{2} L_{1} C_{1}=\frac{\omega_{1}}{\omega_{2}}\left(\omega^{2} L_{1} C_{1}-1\right) \\
& 1-\frac{f_{1}^{2}}{f_{0}^{2}}=\frac{f_{1}}{f_{2}}\left(\frac{f_{2}^{2}}{f_{0}^{2}}-1\right) \\
& f_{0}=\sqrt{f_{1} \cdot f_{2}}
\end{aligned}
$$

If there is a load $R=R_{k}$. Then the values of $L_{1}, C_{1}$ and $L_{2}, C_{2}$ can be found out in terms of $R$ and $f_{1}, f_{2}$.

At the lower cutoff frequency:

$$
\begin{aligned}
& \frac{1}{\omega_{1} C_{1}}-\omega_{1} L_{1}=2 R \\
& 1-\frac{f_{1}^{2}}{f_{0}^{2}}=4 \pi R f_{1} C_{1} \\
& C_{1}=\frac{f_{2}-f_{1}}{4 \pi R f_{1} f_{2}} \\
& L_{1}=\frac{R}{\pi\left(f_{2}-f_{1}\right)} \\
& L_{2}=C_{1} R^{2}=\frac{R\left(f_{2}-f_{1}\right)}{4 \pi f_{1} f_{2}} \\
& C_{2}=\frac{L_{1}}{R^{2}}=\frac{1}{\pi R\left(f_{2}-f_{1}\right)}
\end{aligned}
$$

This completes the design of the prototype band pass filter. Based upon the above formulae, the values for components are as follows:

$$
f_{1}=25.5 \mathrm{MHz} f_{2}=30.5 \mathrm{MHz} R=50 \Omega
$$

Therefore:

$L_{1}=\frac{R}{\pi\left(f_{2}-f_{1}\right)}=3.18 \mu \mathrm{H}$

$C_{1}=\frac{f_{2}-f_{1}}{4 \pi R f_{1} f_{2}}=10.231 \mathrm{pF}$

$L_{2}=C_{1} R^{2}=\frac{R\left(f_{2}-f_{1}\right)}{4 \pi f_{1} f_{2}}=25.57 \mathrm{nH}$

$C_{2}=\frac{L_{1}}{R^{2}}=\frac{1}{\pi R\left(f_{2}-f_{1}\right)}=1.27 \mathrm{nF}$

The simulation of the circuit is done in MULTISIM and the response is obtained. The Fig. 7 shown below is the bode plot of this filter.

From the response shown in Fig. 7, the upper and lower cutoff frequencies are 25.53 and $30.478 \mathrm{MHz}$ respectively. Hence the bandwidth becomes $4.948 \mathrm{MHz}$ which is close to the desired bandwidth. But it does not have the proper attenuation in the stop band.

\section{M-Derived T-Section Filter}

The m-derived or $m$-type filter section is a development of the $k$-type section. The main problem being addressed was the need to achieve a better match of the filter into the terminating impedances. All filters designed by the image method, in general, fail to give an exact match but the m-type filter is a big improvement with suitable choice of the parameter $m$. The m-type filter section has a further advantage in that there is a rapid transition from the cut-off frequency of the pass band to a pole of 
attenuation just inside the stop band. The parameter $\mathrm{m}(0<m<1)$ adjusts the position of this pole of attenuation. Smaller values of $\mathrm{m}$ put the pole closer to the cut-off frequency. Larger values of $m$ put it further away. In the limit, as $\mathrm{m}$ approaches unity, the pole approaches $\omega$ of infinity and the section approaches a k-type section. To overcome the limitations with constant k-type filter, m-derived filter has been designed for the given specifications as follows. Constant $K$-type $t$-section filter can be converted into m-derived t-section as shown in Fig. 8.

where, $Z_{1}$ is the series combination of $L_{1}$ and $C_{1}$ and $Z_{2}$ is the parallel combination of $L_{3}$ and $C_{3}$ of the constant-k type filter. As seen from the Table 2, the band width obtained for $m=0.99$ is closer to the desired bandwidth than the other values of $\mathrm{m}$. Hence, the $m$-derived band pass filter has been designed for $m=0.99$.

The simulation of the circuit is done in MULTISIM and the response is obtained. The Fig. 10 shows bode plot of this filter. From the curve, the upper and lower cutoff frequencies are 25.423 and $30.66 \mathrm{MHz}$ respectively. Hence the bandwidth becomes $5.237 \mathrm{MHz}$ which is close to the desired bandwidth. But it does not have the proper attenuation in the stop band. Hence an improved version of the m-derived filter is discussed in the next section.

Table 2. Range of frequencies for different values of $m$

\begin{tabular}{|c|c|c|c|c|c|c|c|}
\hline \multirow[b]{2}{*}{$\mathrm{m}$} & \multicolumn{2}{|l|}{$\frac{m Z_{1}}{2}$} & \multicolumn{2}{|l|}{$\frac{1-m^{2}}{4 m} Z_{1}$} & \multicolumn{2}{|l|}{$\frac{Z_{2}}{m}$} & \multirow[b]{2}{*}{ Cutoff frequency } \\
\hline & $\mathrm{L}(\mu \mathrm{H})$ & $\mathrm{C}(\mathrm{pF})$ & $\mathrm{L}(\mu \mathrm{H})$ & $\mathrm{C}(\mathrm{pF})$ & $\mathrm{L}(\mathrm{nH})$ & $\mathrm{C}(\mathrm{nF})$ & \\
\hline 0.5 & 0.39750 & 81.848 & 0.00424 & 7.67325 & 51.1400 & 0.3650 & $23.314-33.35$ \\
\hline 0.75 & 0.59625 & 54.5653 & 10.90280 & 2.98404 & 34.0933 & 0.9525 & $24.721-31.36$ \\
\hline 0.9 & 0.71550 & 45.4711 & 30.12600 & 1.07990 & 28.4110 & 1.1430 & $25.26-30.76$ \\
\hline 0.95 & 0.75525 & 43.0770 & 61.96900 & 0.52500 & 26.9150 & 1.2065 & $25.42-30.66$ \\
\hline 0.99 & 0.78705 & 41.3373 & 316.40200 & 0.10282 & 25.8282 & 1.2573 & $25.509-30.569$ \\
\hline
\end{tabular}

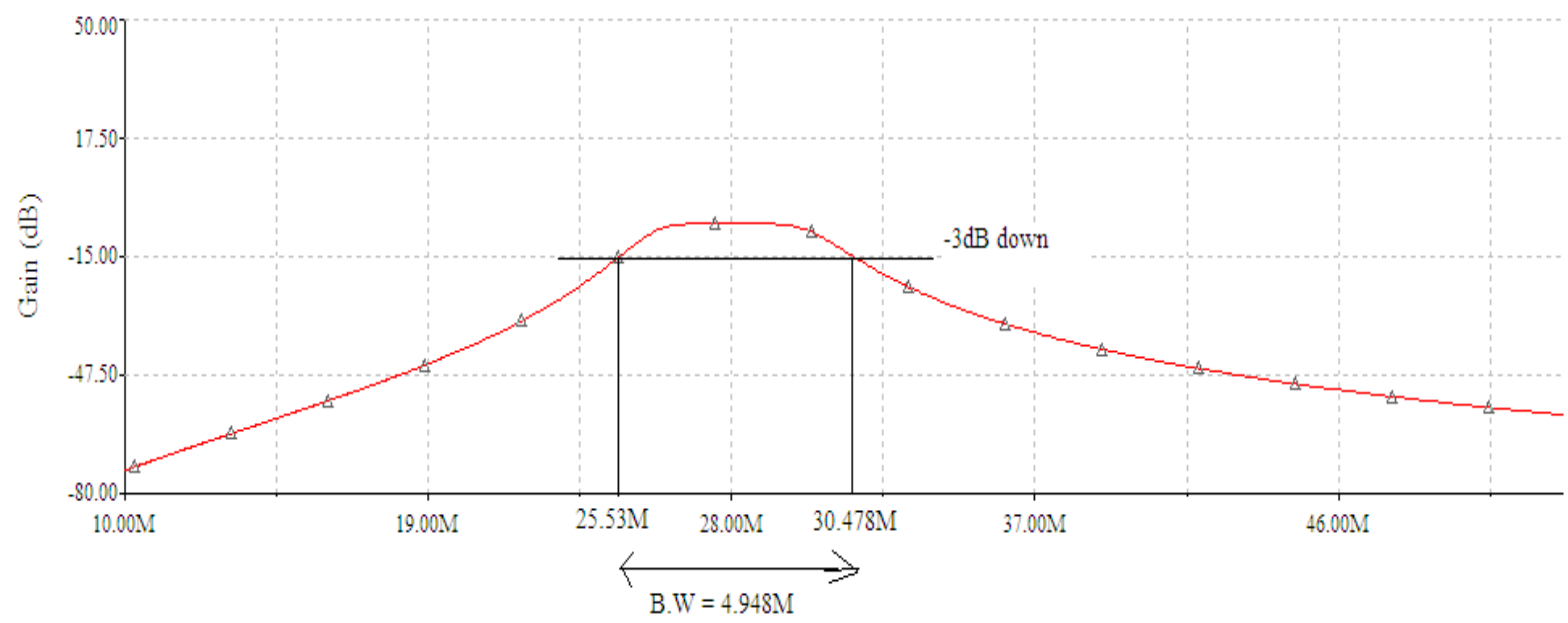

Fig. 7. Response of constant K-type BPF in MULTISIM
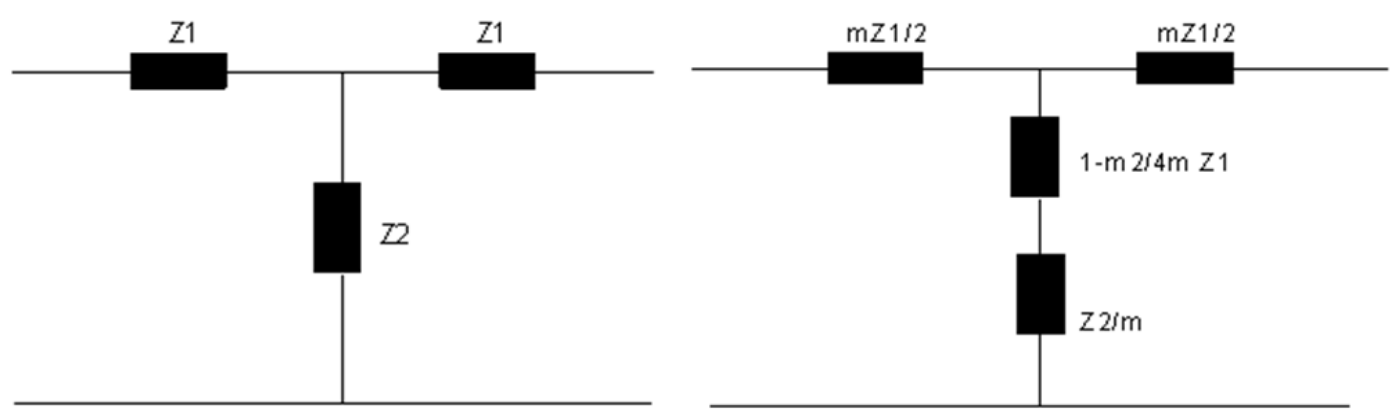

Fig. 8. Constant-k type t-section filter and equivalent m-derived circuit 


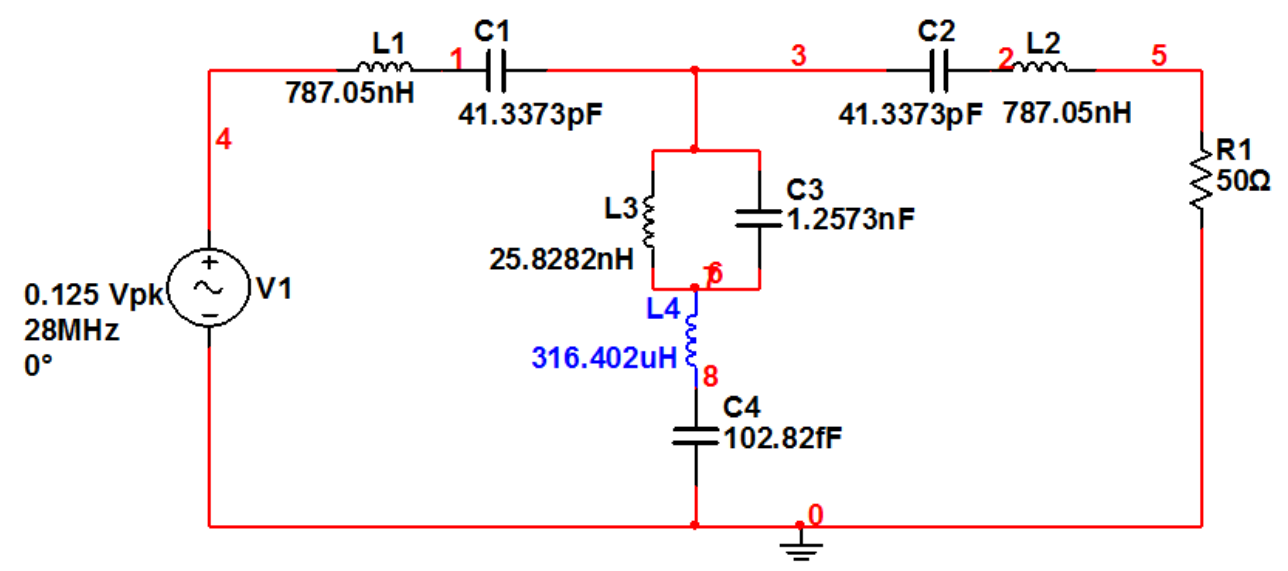

Fig. 9. Shows the circuit of the m-derived filter designed using MULTISIM

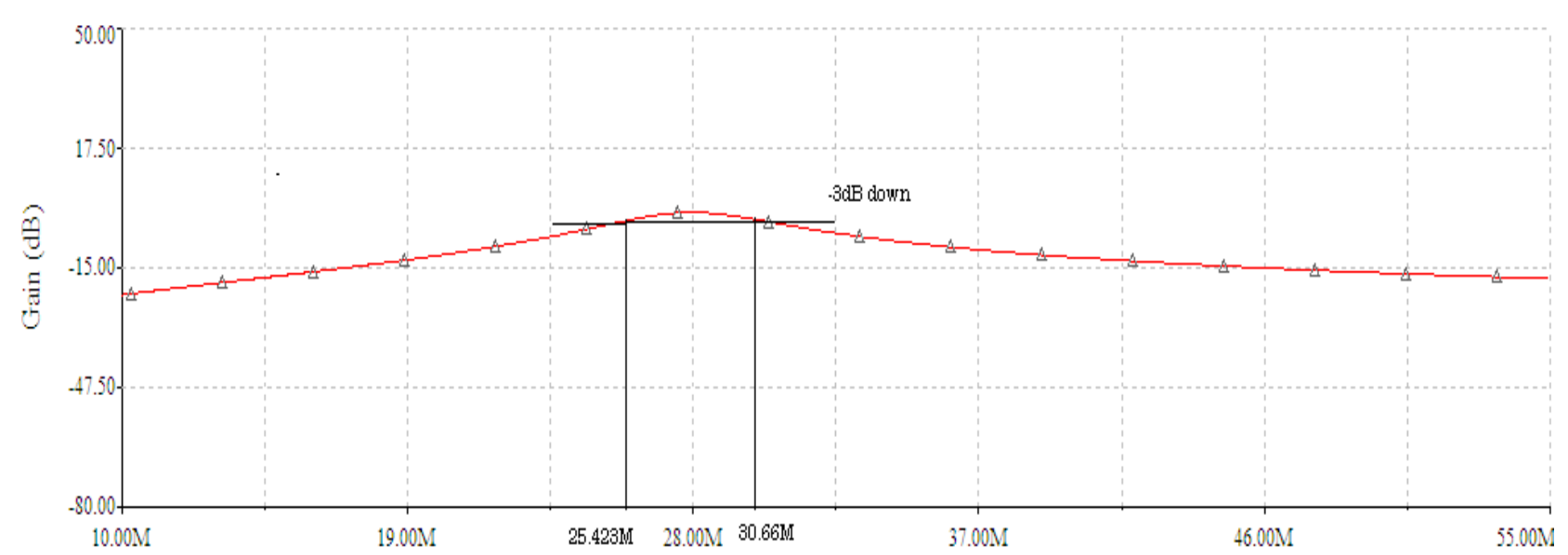

Fig. 10. Response of m-derived filter

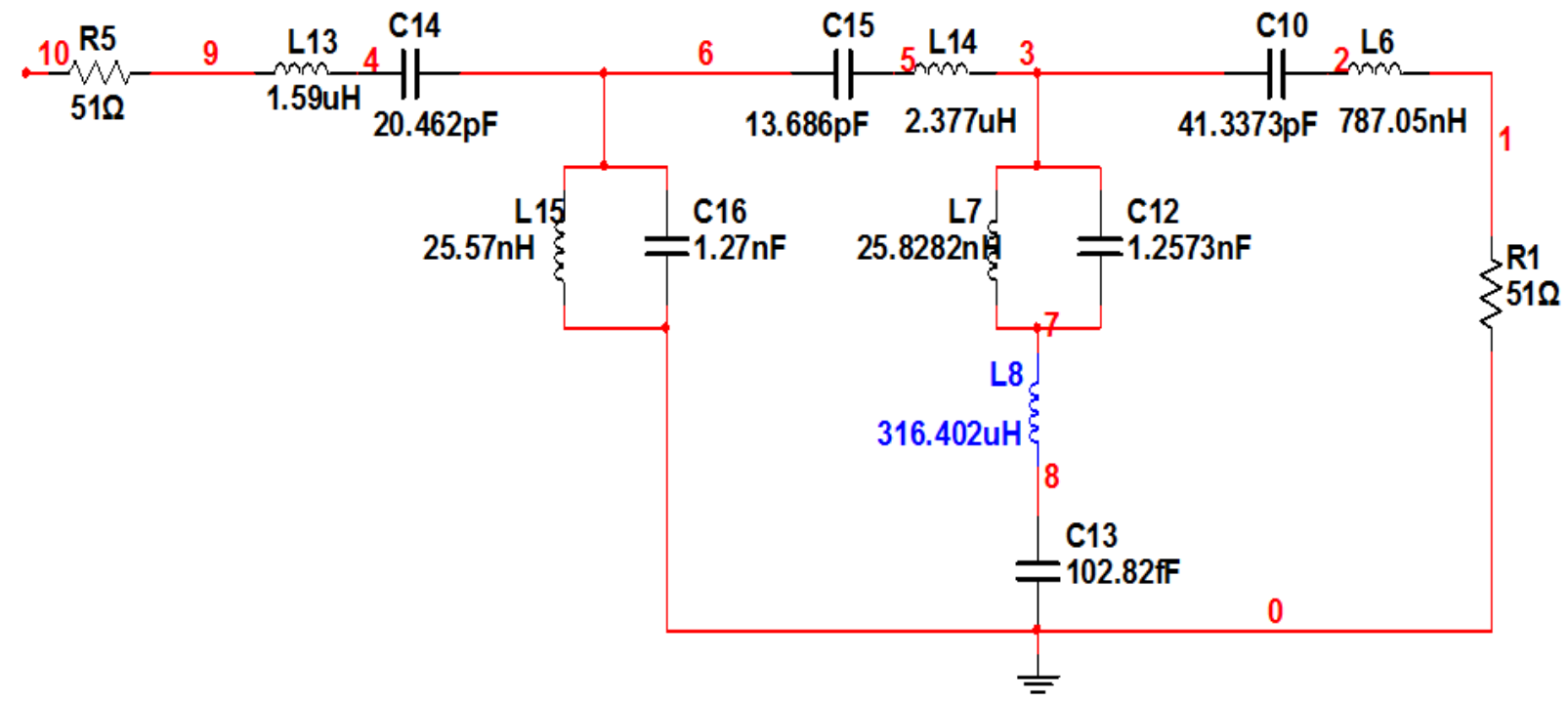

Fig. 11. Composite filter 


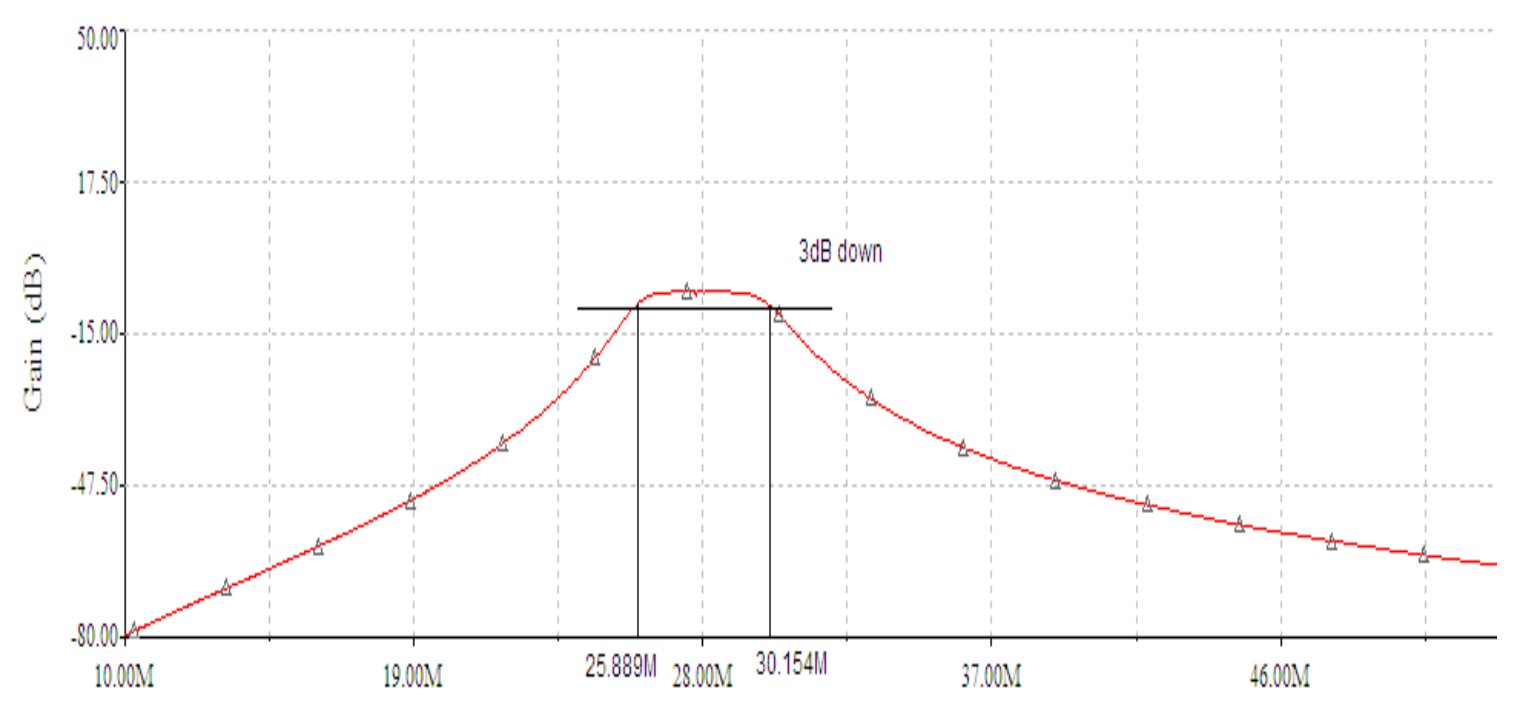

Fig. 12. Response of a composite filter

\section{Composite Filter}

There is a drawback with m-type filters. At frequencies past the pole of attenuation, the response starts to rise again and m-types have poor stop band rejection. For this reason filters designed using m-type sections are often designed as composite filters shown in Fig. 11, using a mixture of k-type and m-type sections and different values of $\mathrm{m}$ at different points to get the optimum performance from both types.

The simulation of the circuit is done in MULTISIM and the response is obtained. The Fig. 12 shown below is the bode plot of this filter. From the curve, the upper and lower cutoff frequencies are 25.889 and $30.154 \mathrm{MHz}$ respectively. Hence the bandwidth becomes $4.265 \mathrm{MHz}$ which is not a desired of bandwidth. But it does have better attenuation in the stop band than the other two filters discussed above.

To further improve the attenuation and the bandwidth, ladder t-section type band pass filter has been designed which is discussed in the next section.

\section{Ladder T-Section Filter}

In image filter design it is common to use topologies that are a modification of the basic ladder topology. These topologies, invented by Otto Zobel, have the same band form as the prototype ladder on which they are based but the transfer function is modified to improve some parameter such as impedance matching, stopband rejection or passband to stopband transition steepness. Usually, the design begins with the simple ladder topology and then some transform is applied to it. The resulting topology is ladder like but no longer obeys the rule that shunt admittances are the dual network of series impedances. The topology invariably becomes more complex and requires higher component counts. Hence, the ladder t-section filter has been designed by cascading two constant-k type filters as shown in Fig. 13 which is designed in MULTISIM.

The simulation of the circuit is done in MULTISIM and the response is obtained. The Fig. 14 shown below is the bode plot of this filter.

From the curve, the upper and lower cutoff frequencies are 25.724 and $30.3 \mathrm{MHz}$ respectively. Hence the bandwidth becomes $4.576 \mathrm{MHz}$ which is close to the desired bandwidth. It has the best stop band attenuation possible of all the filters discussed so far.

\section{Amplifier Filter Board and Hardware Testing}

This section deals with amplifier filter board consisting of whole circuit from amplifier to the low pass filter. The simulation of amplifier filter circuits is done using a software tool called MULTISIM. The circuit diagram of the amplifier filter shown in Fig. 15 have Input signal, Amplifier, Band pass filter, Amplifier and Low pass filter blocks.

The testing of the circuit was done on the hardware part. Source is the signal generator which gives an input signal of $28 \mathrm{MHz}$ and an input voltage of $0.125 \mathrm{~V}$. This input is given to the amplifier selector board which is the actual circuit to be mounted on the seeker. there are three inputs and outputs to amplifier selector board. For lab testing, the three inputs can be used interchangeably and the corresponding outputs measured on the respective pins. But in real time application the three inputs are sum 
signal input, azimuth difference input and elevation difference input and their corresponding outputs. The output of these pins is measured on the spectrum analyzer, for measuring the output in frequency domain and on the oscilloscope for measuring in time domain. The power gain measured with the spectrum analyzer was $5 \mathrm{dBm}$ and the voltage measured with the oscilloscope was $1.68 \mathrm{~V}$.

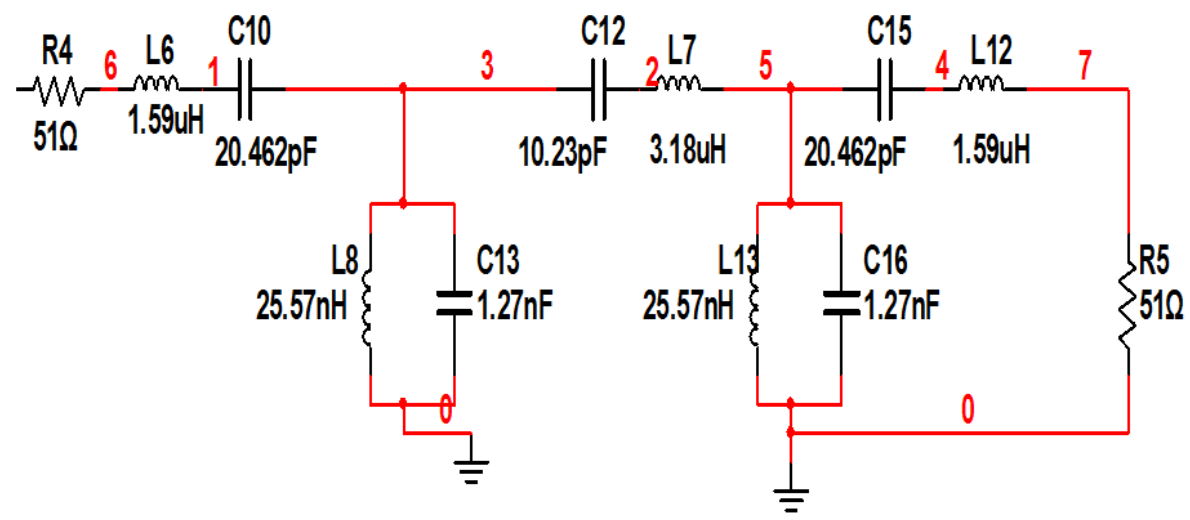

Fig. 13. Ladder t-section filter

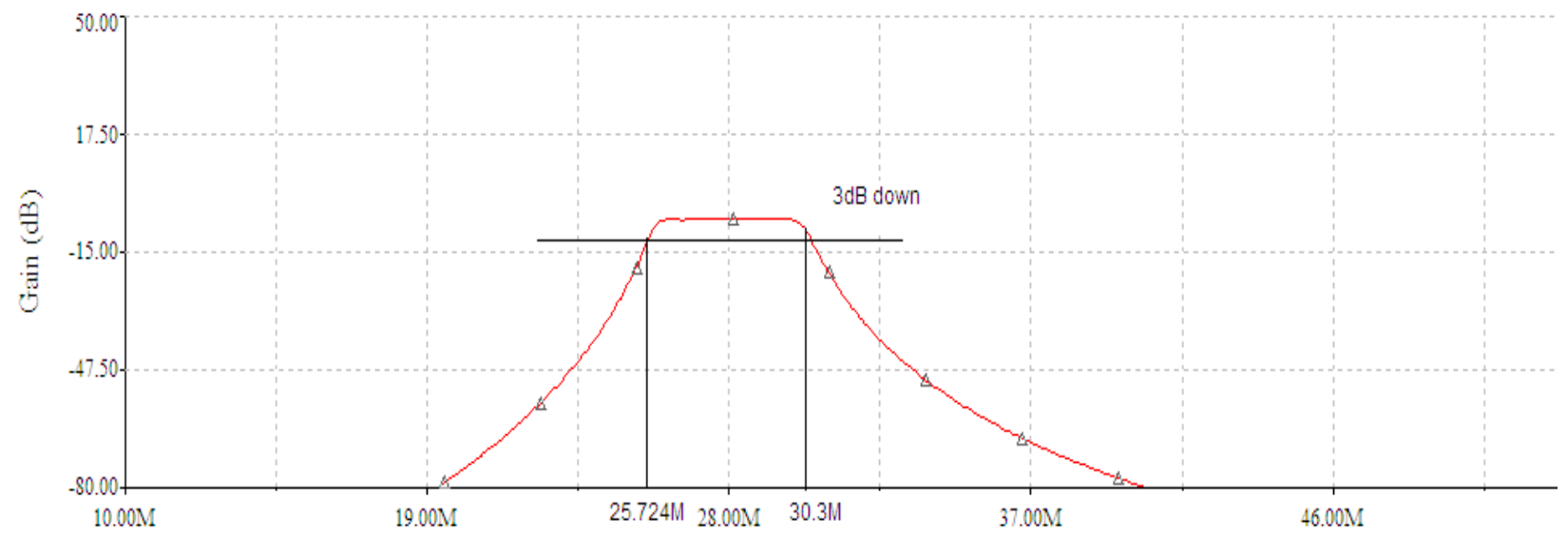

Fig. 14. Response of a ladder t-section filter

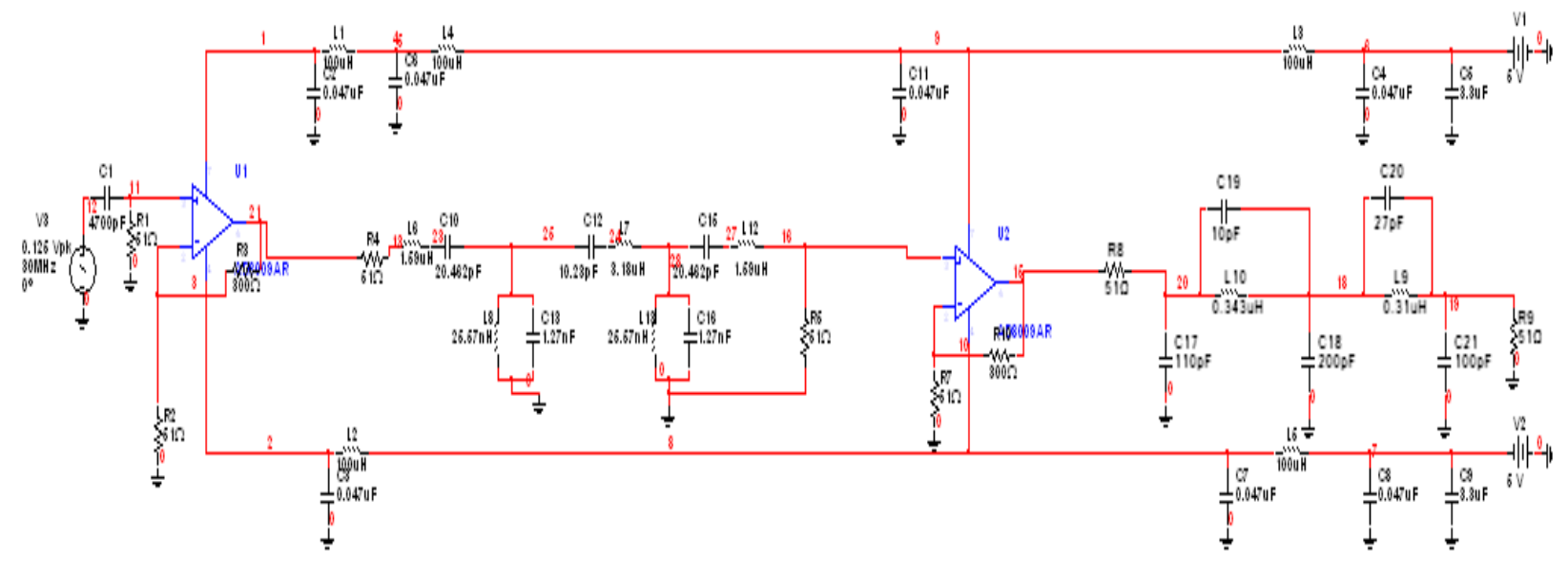

Fig. 15. The complete circuit diagram (in MULTISIM) 


\section{Results}

This section deals with the results of simulation and hardware board testing. The simulation was done in the software tool called MULTISIM and the hardware testing was done using signal generator, spectrum analyzer and oscilloscope. It was seen in previous section that we had designed various band pass filters wherein each was an improvement over the other with the ladder t-section being the best of the lot with better attenuation in the stop band and better bandwidth. The graph in Fig. 16 shows a comparison response of each of the band pass filters.
The photographs shown below were taken during testing. They show the various instruments used and their respective readings. Figure 17 shows the amplifier selector board on which the complete circuit has been mounted. As can be seen, there are three circuits put on the board each having an input and an output. The gain of $8 \mathrm{~dB}$ was obtained with this amplifier filter board measured using spectrum analyzer. Figure 18 below shows the signal generator with the given input. Figure 19 and 20 below shows the output on the oscilloscope and output measured on the spectrum analyzer.

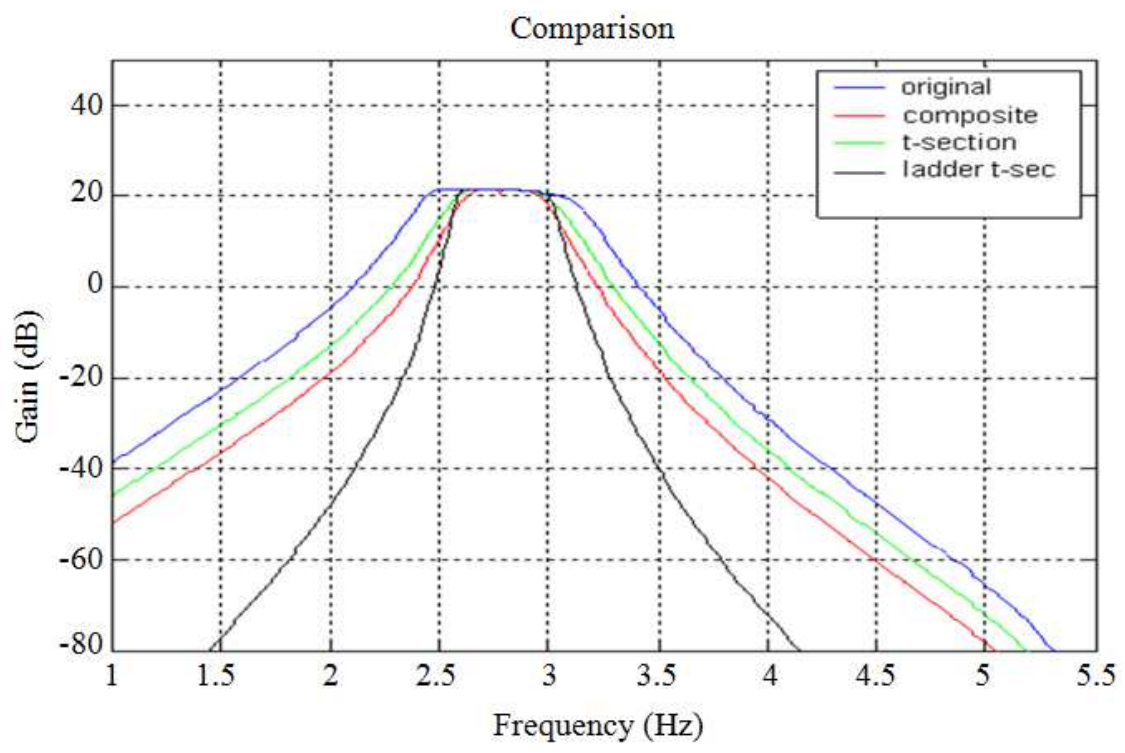

Fig. 16. Comparison of all BPF with amplification

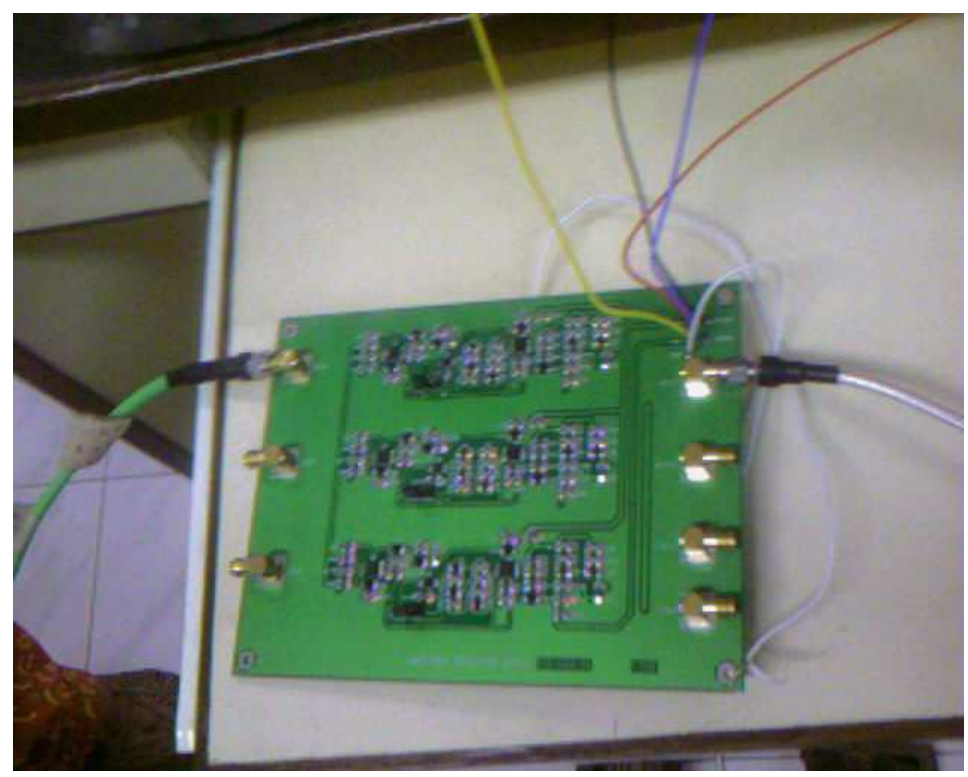

Fig. 17. Amplifier selector board 


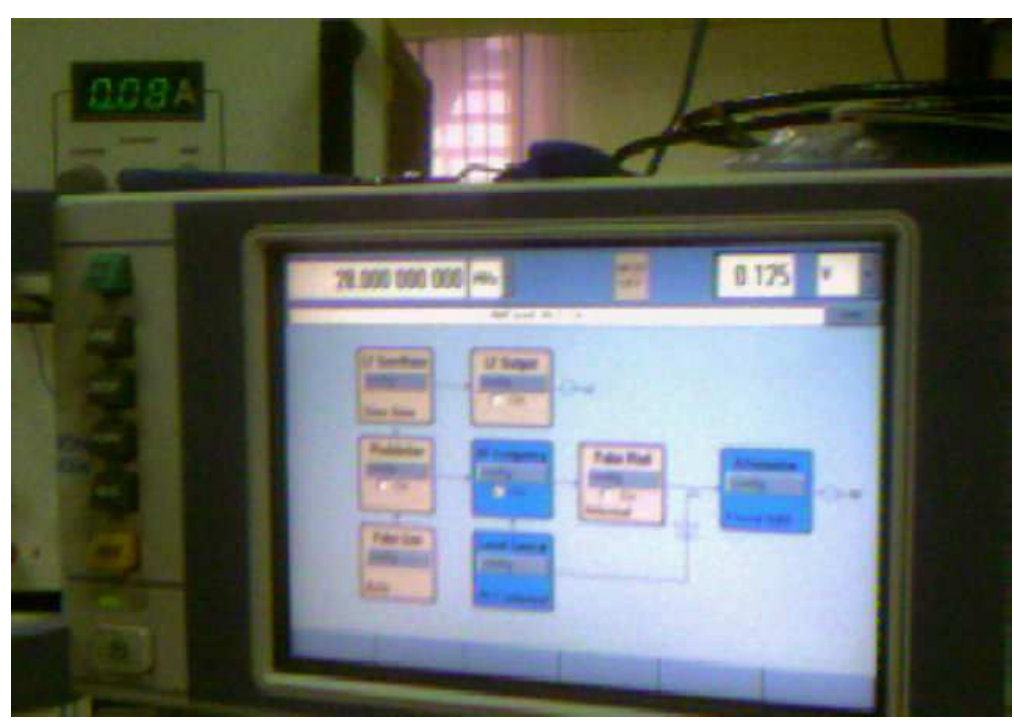

Fig. 18. Signal generator

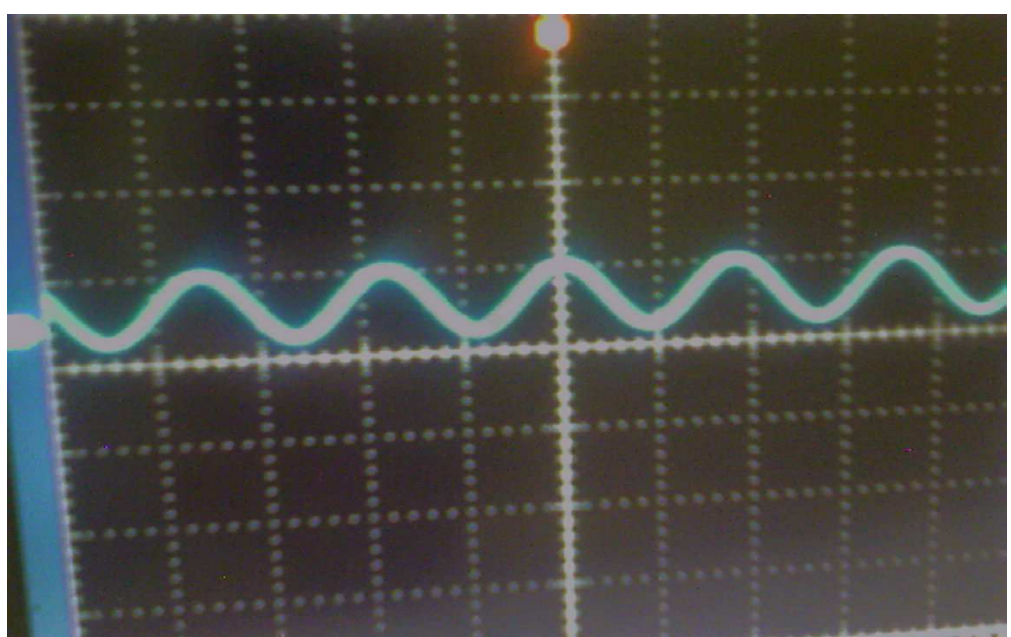

Fig. 19. Output observed in oscilloscope

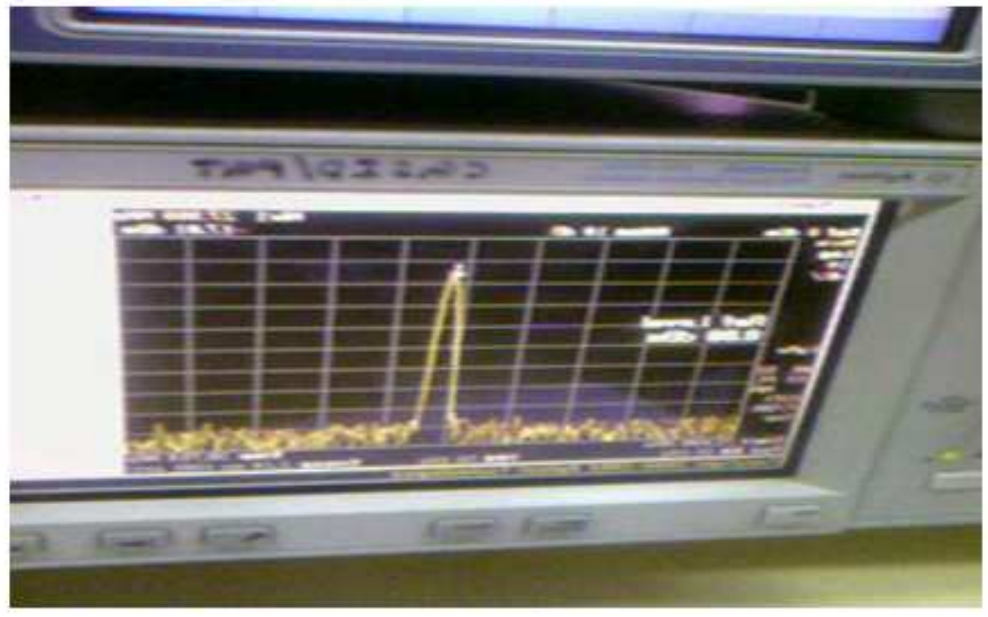

Fig. 20. Output in spectrum analyzer 


\section{Conclusion}

The realization and simulation of the amplifier and filters has been done in MULTISIM and testing is done on the hardware part as well. During testing the gain obtained from this two stage amplification amplifier filter board was $8 \mathrm{~dB}$. Constant-k type, mderived type, composite filter and ladder type Tsection band pass filters have been designed of which the stop band attenuation and bandwidth of the designed Ladder T-section band pass filter are better in comparison with the realized band pass filter and other designed filters. The on-board radar designed is for only air-borne applications (Tahim et al., 2003). More over precision of these radars is not sufficient to hit and crush, so the rocket itself is provided with onboard radar to track the target, block it buzzing around and pulverize.

\section{Acknowledgment}

We thank DRDL, Hyderabad for permitting us to utilize the labs to complete the on-board radar system filter design and test the working model.

\section{Author's Contributions}

N Dinesh Kumar: Designed and tested composite filters and ladder t-section filter and wrote the paper.

R Divya Ravali: Designed and tested low pass filter and band pass filter. Performed analysis of ladder Tsection filter.

Panchavati R. Srirekha: Designed and tested $\mathrm{m}$ derived filter. Performed analysis of $\mathrm{m}$-derived and the final filter circuit design.

\section{Ethics}

This article is original and contains unpublished material. The corresponding author confirms that all of the other authors have read and approved the manuscript and no ethical issues involved.

\section{References}

Bao, Y., Z. Shi and K. Chen, 2010. Intermediate frequency circuit design for a $60 \mathrm{GHz}$ LFMCW radar. Proceedings of the International Conference on Microwave and Millimeter Wave Technology, May 8-11, IEEE Xplore Press, Chengdu, pp: 270-273. DOI: 10.1109/ICMMT.2010.5524927

Skolnik, M.I., 1990. Radar Handbook. 2nd Edn., McGraw-Hill, ISBN-10: 007057913X, pp: 1163.

Stephens, J.P., 1996. Advances in signal processing technology for electronic warfare. IEEE Aerospace Electronic Syst. Magaz., 11: 31-38. DOI: $10.1109 / 62.544024$

Tahim, R.S., J.J. Foshee and K. Chang, 2003. Phasedarray radar for airborne systems. Proceedings of the SPIE 5071, Sensors and Command, Control, Communications and Intelligence (C3I) Technologies for Homeland Defense and Law Enforcement II, (DLE' 03), SPIE.

DOI: $10.1117 / 12.487522$ 